water instead of, as reputed, 25 . Let us therefore call them all 22 drops. Now the quantities of serum actually present in the tubes are in volumes respectively 10/11,5/11.2/11, $1 / 11$, and 0 of a water-drop. And 10/11 of a drop in 22 drops is precisely 1 in 24.2 instead of the reputed 1 in 25 . Similarly for the other tubes, each of which also possesses an error of about 3 per cent. in the reputed dilution of the serum. The worker has actually put up dilutions of 1 in $24.2,1$ in 48.4 , \&c., but has been deluded into calling them 1 in 25, 1 in 50, \&c. Of what conceivable importance can this be when in the course of active enteric infection we are dealing with changes of titre in the serum amounting to anything from some hundreds per cent. up to thousands per cent. ?

Thus far we have succeeded in reducing alleged errors (in technique), which if cumulative amount to 70 per cent., to a single error (in nomenclature) of about 3 per cent.

(c) The "Directions" make no adequate reference to any of Donald's "six precautions," nor indeed to any other precautions we have taken in connexion with this matter. To point this out is rather a compliment than a criticism when viewed in the proper light, though apparently it was not so intended. But on this occasion we may briefly consider some of these precautions.

1. The outer diameter of the pipette nozzle. The great, importance of variations in this measurement and the relative unimportance of the inner diameter were fully recognised. Close gauges for the outer diameter of the nozzle were provided from the first. In view of what has already been said about the manufacture of the pipettes the matter may be left there for the moment.

2. The practical vertical position of the pipette nozzle. If there is any reason to believe that the pipette is not held vertically (or nearly so) by the worker, Donald performs a service in drawing attention to the omission of instruction on this point. It did not seem probable to us that anyone would be likely to attempt to fill in drops from a teat pipette into very narrow tubes placed in rows in a stand except with the pipette held vertically or nearly so. After watching many successive classes of students learn the method I should not have thought the danger very great. Certainly the vertical position is the usual one and the natural one, and something must always be left to the intelligence of the operator. Moreover, whatever position the worker adopts, he is likely always to assume pretty much the same position, so that his drops will all have similar errors. Still the lack of reference to the position of the pipette is a distinct omission which can easily be remedied, and thanks are due to Donald for calling attention to it. Unfortunately, the rest of his criticisms are not of the same value, and some of them are merely trivial.

3. He disapproves of hand-pipettes with teats because the drop-size varies somewhat with drop-rate. By using wide differences in drop-rate-namely, from 1.3 seconds to 10 seconds per drop (nearly an eight-fold increase in drop-time) -he obtains a difference in drop-size of just over 5 per cent. His lower limit of time is quite excessive for this kind of drop-measurement. But even from his own figures one might safely have hazarded the opinion that any differences in droptime likely to be present when a worker is filling in tubes " by gentle pressure on the teat " of a properly handled pipette in the earnest pursuit of his rontine work are unlikely to amount to 1 per cent. And we should willingly have presented Donald with a 1 per cent. error here did we not happen, as a matter of fact, to know from many trials that with a Drejer pipette the drop-rate error does not nearly reach this figure.

4. The temperature. We are charged with ignoring in the present technique the effect of temperature on dropmeasurement. The charge is perfectly well founded, as would also be a charge that we wilfully ignore the effect of differences in the barometric pressure and in the relative humidity of the atmosphere, and give no correction for the variation in the force of gravitation at different points on the surface of the globe, though all these phenomena have some effect on our operations. It is simply a question of order of magnitude. As regards the temperature, each rise of $1{ }^{\circ} \mathrm{C}$. in temperature lowers the surface tension of water about $\frac{1}{4}$ per cent., and consequently also lowers the dropvolume. Thus, a difference of temperature of $8^{\circ} \mathrm{C}$. $\left(14^{\circ}\right.$ to $15^{\circ} \mathrm{F}$.) will make a difference of 2 per cent. in the dropvolume of water. But Donald forgets to point out that the drop-volume of serum and of the standardised agglutinable cultures are altered at the same time in the same direction, so that their ratios can only be affected to an entirely insignificant extent per $1^{\circ} \mathrm{C}$. It follows that unless the worker has it in mind to carry out his manipulations in a refrigerator or in a Turkish bath-and for this we have failed to provide-the factor of temperature is entirely negligible.

It would be tedious, and I think unnecessary, to pursue this discussion further. I have attempted to show that the whole framework of the criticism put forward by Donald rests on a totally inadequate foundation, and that he was misled in supposing himself to have discovered errors of such gravity in Dreyer's method that it became a "public duty" to point out its inaccuracy.

It was a priori unlikely that a responsible and authoritative body like the Medical Research Committee would have undertaken the issue of standardised agglutinable cultures, and strongly supported the use of the technique in question by supplying the agglutination sets so widely as they have done had the method been ill-devised, inascurate, and slip-shod.

It appears, on the contrary, that the most recent scrutiny, and one conducted by an expert in drop-measurement, has failed to prove it open to serious criticism in any significant detail. It is the objections offered which are seen to be illfounded. But though it is proper that inaccurate criticism should be firmly rebutted, it must not be supposed that anything said here in meeting Donald's objections is meant to throw doubt on the sincerity of his intentions. It was unfortunate that he failed to explain more clearly that the supposed error of 40 per cent. was not only entirely tactitious, but also one already carefully precluded. It was still more unfortunate that such unsubstantial criticisms should appear under the name and ægis of an important institute.

\section{A NEW REMEDY FOR SYPHILIS, LUARGOL OR "102."}

\section{BY N. S. BONARD, M.D.,}

SURGICAL OFFICER, LONDON LOCK HOSPITAL: LATE SENIOR HOUSE SURGEON, FRENCH HOSPITAI, LONDON.

Since the introduction of salvarsan, several substitutes have been put on the market. In 1913, Mouneyrat in France discovered galyl or tetraoxydiphosphotetraminodiarseno. benzol. He added two phosphoric groups to arsenobenzol, and made a compound more powerful in arsenic than neosalvarsan. Dr. Danysz, of the Institut Pasteur in Paris, who has been working for a long time on the treatment of trypanosomiasis, introduced two years ago a new compound as a substirute for salvarsan called "102," or luargol, in which he added silver salts and antimony to arsenobenzol.

The antiseptic properties of silver are well known. For many years the silver nitrate pencil has been used as canstic and for treating syphilitic mucous plaques. These properties have again proved their value when colloidal silver prepara. tions were used as ointments or intravenous injections in septic conditions. Charcot has shown the perfect tolerance of silver, and that poisonous symptoms appeared only after absorption of 30 grammes of silver nitrate. Danysz at first tried solutions of silver nitrate. He noticed that at the 1 in 80,000 strength this solution had not a great microbicidal action, and had the grave disadvantage of causing venous thromhosis. He then combined silver with eosin, and obtaioed a precipitate which dissolved by adding sodium chloride. This product proved to have a powerful antisyphilitic action in experimental treatment on rabbits. But the veins were still considerably altered by this product, which could not be used at all in therapy.

After further research, Danysz tried to combine silver with dioxydiaminoarsenobenzol, and obtained another compound. A solution of silver nitrate was mixed with an aqueous solution of dioxydiaminoarsenobenzol hydrochloride. A more or less brown clear solution, varying with the quantity of silver used, was obtained. By adding to this sodium chloride, a flocculent precipitate was formed and no modification appeared in the liquid. This precipitate treated with sulphuric acid gave another abundant paleyellow precipitate and the liquid contained only traces of arsenic and silver, which demonstrated that silver and chlorine had been fixed by dioxydiaminobenzol. Danysz had then the idea of replacing chlorine, which is without any therapeutic property, by bromine or iodine, which have 
microbicidal properties. He obtained thus a bromo-silver arsenobenzol, which he called the " 88 " 2 . This product was very satisfactory and experience proved its efficiency. Hoping further to increase the specific action, Danysz tried to introduce in this " 88 " 2 a certain quantity of antim'sny as trichloride of antimony $\left(\mathrm{SbCl}_{3}\right)$. He then obtained a dioxydiaminoarsenobenzolstibicosilver sulphate, whose formula is : $\left(\mathrm{C}_{2} \mathrm{H}_{12} \mathrm{O}_{2} \mathrm{~N}_{2} \mathrm{As}_{2}\right) 2 \mathrm{AgBrSbO}\left(\mathrm{H}_{2} \mathrm{SO}_{4}\right)_{2}$, and which Danysz called luargol, or "102." In analysis the following is found :-

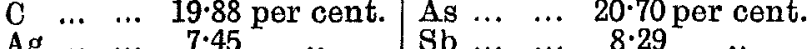

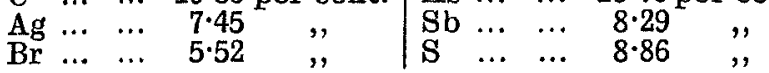

Silver is fixed in this compound as a bromide, antimony as antimonyl. The addition of a silver salt increases the antiseptic properties of arsenobenzol. Bromide has been chosen because experience proved that the combinations with silver bromide are more active than those made with chloride or nitrate and less toxic than those with silver iodide. The addition of antimony seemed indicated in those cases of syphilis and trypanosomiasis in which arsenic had no effect at all, and in which antimony proved very efficacious.

Danysz's experience shows that the " 102 " is more active than any other arsenical compound in trypanosomiasis. For instance, in rabbits infected with surra, the evolution of the disease is about the same as in trypanosoma infections in man. After an incubation period from 5 to 7 days, there are raised temperature and wasting. Thirty to fifty days following inoculation lesions appear on the skin and mucous membranes; later there are nervous troubles. If treatment is begun during the incubation period all arsenical compounds cure the animals easily. If treatment starts during the second period, atoxyl, arsenophenylglycine and arsenobenzol give only a certain proportion of cures, while " 88 " 2 and "102" cure them all During the last period, when lesions have appeared, " 102 " alone could cure nearly all animals (5 in 6 ) with doses of $0.01 \mathrm{cg}$. per kilogramme. For obtaining the same result with " 88 " 2 twice as much had to be given. Other arsenical compounds at the same doses gave no result at all. Thus, the relation between the tolerated dose and the curative dose of luargol is 80 to 1 , for trypanosoma gambiense 100 to 1 , while for the same trypanosomiasis, the relation for atoxyl is 4 to 3 , arsenobenzol 10 to 1 , arsenophenylglycine 3 to 1 .

In sleeping sickness luargol is 75 times more active than atoxyl; 30 times more active than arsenophenylglycine and 10 times more active than arsenobenzol. Experience has shown also that luargol cured mice infected with Trypanosoma dimorphon which is known to be resistant to arsenobenzol, atoxyl and phenylglycine. In rabbits the treponemata in the chancre disappear in 24 hours with doses of 0.002 to $0.003 \mathrm{gm}$. of luargol per kilogramme. The same result is obtained in men after a single injection of 0.10 or $0.15 \mathrm{gm}$. The same, of course, is seen with arsenobenzol, but with much larger doses. Luargol can thus be used in cases where arsenobenzol is not tolerated.

Luargol is interesting as a new substitute for salvarsan because the effective therapentic dose is far off the toxic dose as compared with other arsenical compounds. Very small doses need to be injected for obtaining a therapeutic effect. With doses of 0.10 or even $0.05 \mathrm{gm}$. the therapeutic effect is already evident, while the toxicity is about $0.20 \mathrm{gm}$. per kilogramme in rabbits. The injections are given intravenously in the same manner as employed in salvarsan or neosalvarsan. They are repeated every second or third day, the first dose to be injected being 0.10 or $015 \mathrm{gm}$. The following doses are increased up to 0.30 or $0.35 \mathrm{gm}$. The total dose of 1.0 to 1.20 or perhaps $1.50 \mathrm{gm}$. ought to be injected in 6 or 7 injections.

After the first injection, there is often a febrile reaction more or less intense which is never serious and disappears in a few hours without any serious vomiting, headache, or gastro-intestinal troubles. Generally, the further injections give rise to no reaction. The slight reaction after the first injection is probably due to the destruction of the spirochætes. The intensity depends upon the general state of the patient and is variable. In strong subjects in good condition, there is very often no reaction at all. In three cases out of 500 treated with luargol at the Hôpital Cochin, in Paris, on the day following the first injection, there was a general scarlatiniform rash with a moderate temperature.
This rash disappeared in two days without any further trouble. This is the only unusual incident registered up to the present time.

The immediate therapeutic effects of luargol are about the same as those obtained with arsenobenzol with doses three times as strong. The primary, secondary, and even the tertiary symptoms yield in a few days with doses of luargol always much smaller than those which would be required with salvarsan, neosalvarsan, or any other substitute. In 80 per cent. of the cases treated the Wassermann test gave a negative result one munth after the beginning of the treatment, and after 1.0 to $1.20 \mathrm{gm}$. had been injected. But here, as in the treatment with salvarsan, the Wassermann test can give again a positive result after a few months. Another course of injections may then become necessary. All these cases have been treated without mercury or local treatment. In every case luargol has proved to be a powerful tonic, the general state of patients improving quickly. Luargol is very stable and keeps in solution without alteration for many hours. A great number of patients can be injected with the solution. The treatment seems to be without any danger in ordinary cases. The results are very rapid, as the injections can be repeated every second or third day.

Luargol is a lignt yellow-orange powder insoluble in water. It is soluble in a solution of sodium hydrate, $0.40 \mathrm{cg}$. of the latter being able to neutralise $1 \mathrm{gm}$. of luargol. Practically the best way to proceed to get the solution is to dissolve $1 \mathrm{gm}$. of the powder in 15 c.c. of a 4 per cent. solu. tion of sodium hydrate. The powder dissolves quickly, and a dark-brown, nearly black, thick solution is obtained. The injections can be made by the syringe with concentrated solutions, in the same way as with neosalvarsan by Ravault's method; in this case a 1 per cent. solution is prepared by adding distilled water up to 100 c.c., each c.c. containing $0.01 \mathrm{gm}$. of luargol. If the glass-tubing apparatus is preferred, the solution must be 1 per 1000, and made by adding normal saline up to 1000 c.c. Each 10 c.c. will contain $0.01 \mathrm{gm}$. of the drug. In both cases the solution is filtered. This way of proceeding is very convenient for hospitals where a great number of injections are made at the same time. The preparation is supplied in sealed tubes containing $1 \mathrm{gm}$. for hospital purposes, or in five different strengthsviz., $0.10,0.15,0.20,0.25,0.30 \mathrm{gm}$. Complete outfits with a phial of the alkaline solution, a filter, \&c., with full instructions for use, are supplied also for private cases.

Posology of "10\%."-In acquired syphilis with primary, secondary, or tertiary symptoms without nervous complications, the following doses should be injected in a normal adult patient:- $0.15,0.20,0.25,0.30,0.30,0.30 \mathrm{gm}$, i.e. totally $150 \mathrm{gm}$. in six injections repeated every second, third, or fourth day. For a female patient in the same condition the following doses: $0.10,0.15,0.20,0.25,0.25$, $0.25 \mathrm{gm}$., i.e. totally $1.20 \mathrm{gm}$. In cases with nervous or any other complirations the treatment should be carried on with quite small doses increased very slowly, if the first dose is well tolerated. The injections are made into the veins exactly in the same manner as is employed with any other drugs. If a concentrated solution is used with the syringe, the injection is to be marle very slowly into the vein, especially with doses over $015 \mathrm{gm}$. No food should be taken for four hours previous to the injection. The patient must rest for a few hours afterwards. He can have a cup of tea, but no food is to be allowed until four or five hours after the injection, when a light meal may be taken. It is better to make the injection about 5 or 6 P.M., as then the patient can rest until the following morning.

Contra-indications. - The contra-indications for luargol are the same as for other arsenical compounds, i.e. asystoly, uræmia, and a cachectic state not due to syphilis. In badly compensated heart disease and in syphilitic affections running an acute evolution, such as acute meningitis, nephritis, jaundice, \&c., the increasing doses should be very small, 0.006 to $0003 \mathrm{gm}$. There are very few absolute contraindications, as luargol is always well tolerated and seems to be harmless in uncomplicated cases.

Clinical results. - During the past six months, through the courtesy of Mr. A. Shillitoe and Mr. C. Gibbs, of the honorary staff of the Lock Hospital, I have treated about 100 cases of syphilis at different stages with luargol. A certain number of cases have been treated as out-patients at the French Hospital. All these cases, without exception, have done well and the results are most satisfactory. The therapentic effects have 
been more rapid than with satvarsan, neosalvarsan, galyl, novarsenobenzol, or neokharsivan in every case. No serious complications or reactions have been observed. All symptoms of the disease have cleared up rapidly. The injections have been made with concentrated solutions of " 102 " by means of a 20 c.c. glass syringe. All out-patients have been treated in the consulting-room and have left directly afterwards. In some cases they did not even strictly follow the instructions given, but no harm appears to have resulted. In the wards of the Lock Hospital the patients were not kept in bed after being injected. Frequent small doses have proved to be of greater benefit and to give better results than larger doses at longer intervals. Luargol alone has been given in every case and no local treatment made. The cases herein reported are chosen at random and contain only clinical results.

CASE 1.-The patient, a young man aged 23, had been exposed to infection at the beginning of February, 1916. Came under treatment on March 1st with a typical syphilitic chancre on the penis: dirty greyish sore with indurated base. Glands in both groins much enlarged; Wassermann test positive.

Treatment.-March 4th: Chancre the size of a shilling. piece, with indurated base and much swelling of prepuce. A dose of $0.10 \mathrm{gm}$. of luargol was given intravenously; no reaction followed. March $7 \mathrm{th}$ : Third day. Swelling of prepuce had gone down; chancre red and clean; epidermisation beginning on the edges. Second dose of $0.20 \mathrm{gm}$. was given; no reaction followed. March 11th: Seventh day. Chancre healing; induration of base nearly disappeared; a small area only not epidermised. Patient feels much better. Third injection of $0.25 \mathrm{gm}$. was given; no reaction followed. March 14 th: Tenth day. Chancre quite healed; induration of base gone; glands in groins much smaller. Patient feels very well. Four further doses of luargol of $0.25 \mathrm{gm}$. were given at regular intervals to complete the treatment.

In this case the chancre was healed in ten days after injecting a total dose of $0.55 \mathrm{gm}$. Up to the present time (August 28th, 1916) the patient, whom I see regularly, has not developed any secondary symptoms. No local treatment was done and no mercury was given.

CASE 2.-The patient, a man aged 32, had been exposed to infection in January, 1916. A syphilitic chancre developed 25 days later. He was treated privately with medicine and applications of boric vaseline without amelioration. Came under my treatment on March 1st. The patient was feeling very ill, giddy, and weak. He complained of headache. Large dirty greyish discharging sore on penis with indurated base, the width of a two-shilling niece; much swelling of prepuce. Glands much enlarged in both groins. Maculopapular rash on chest, back, and limbs. Sore throat, tonsils enlarged, inflamed with white patches. Mucous plaque on under lip. Swallowing difficult and painful. Wassermann reaction positive.

Treatment.-March 1st: Luargol 0.15 gm. injected; slight reaction followed ; temperature $101^{\circ} \mathrm{F}$. for two hours. chancre looked clean and red; on the edges epidermisation could be seen starting. Throat better; the white patches had gone, tonsils less inflamed, swallowing easier; headache the same. Second injection of $0.25 \mathrm{gm}$. given; no reaction followed. March 7 th : Seventh day. Chancre healing, level with the skin, about balf its previous size; induration softer and going down; swelling of prepuce gone. Glands in groins smaller, rash fading and disappearing. Throat much better, tonsils smaller, swallowing quite comfortable without any more pain. Patient felt strong and "fit"; headache very slight. Third dose of $0.25 \mathrm{gm}$. ; no reaction followed. March Ilth: Eleventh day. Only a very small area of the chancre had not healed; induration of base had gone. Glands in groins smaller. Throat quite well. Rash had cleared up, only a few fading spots could still be seen. No further headache. Fourth injection of $0.30 \mathrm{gm}$. of luargol given; no reaction afterwards. March 14th : Fourteenth day. Chancre quite healed, flat with the skin. Rash quite of patient very good. Two further injections of $0.30 \mathrm{gm}$. were given to complete treatment up to $1.55 \mathrm{gm}$. of luargol. June 1st: Seen again; patient quite well; no other symptoms had developed.

CASE 3.-The patient, a girl aged 18, was admitted into hospital with a large typical syphilitic chancre on fourchette, the size of a shilling-piece, with a dirty greyish discharging base. Tissue all around infiltrated, and huge swelling of labia. She felt weak, giddy, depressed; general state bad. Glands in both groins enlarged and Wassermann reaction positive.

Treatment.-First day: Intravenous injection of concentrated colution of "102" by means of syringe; $0.10 \mathrm{gm}$. given at 10 A.M. ; slight reaction. Temperature at 6 P.M. $101^{\circ} \mathrm{F}$., no sickness or headache. At 8 P.M. temperature $100^{\circ}$; at 10 P.M. normal; no rise afterwards. Fourth day: Swelling of labia greatly diminished. Chancre smaller, red and healthy; epidermisation seen growing from the edges. Second injection of $0.15 \mathrm{gm}$.; no reaction afterwards. Fifth day: Swelling gone; chancre half its previous size. Patient feeling stronger and better. Sixth day: Chancre healing quickly; infiltration of base nearly gone; glands in groins smaller Seventh day: Chancre nearly healed; only a small area not epidermised ; sore flat with the skin; infiltration of base gone. Third injection of $0.20 \mathrm{gm}$; no reaction afterwards. Ninth day: Chancre healed; glands smaller; patient feeling very well. Three further injections of $0.25 \mathrm{gm}$. at regular intervals up to the total dose of $1.20 \mathrm{gm}$. luargol was given: no reaction followed the injections.

CASE 5.-The patient, a woman aged 42, had the first symptoms of syphilis seven years ago; was treated privately with medicine and pills. When admitted into hospital for treatment there were several mucous tubercles of different sizes on vulva, and a large ulceration, the size of a two-shilling piece, stretching from right labia to fourchette; base indurated and infiltrated. Both labia much swollen. Glands in groins hard and enlarged, glands in neck. Ulceration, the size of threepenny-piece, on the nose. Throat very sore for last three weeks; tonsils much enlarged and inflamed with ulcerated white patches; swallowing difficult and painful. Six gummata discharging pus, on scalp, the size varying from a sixpenny to a shilling piece. General state of patient very bad. She complained of headache, weakness, giddiness, with pains in the bones; Wassermann reaction positive.

Treatment.-First day: Intravenous injection of concentrated solution of " 102 " at 10 A.M. by means of syringe; dose, $0.10 \mathrm{gm}$. At 2 P.M. temperature normal; at 4 P.M. $99^{\circ} \mathrm{F}$., headache, slight sickness. At 6 P.M. temperature $100.2^{\circ}$; headache the same, sickness worse. At 8 P.M. temperature $99 \cdot 1^{\circ}$, headache the same, sickness better; at 10 P.M. temperature normal, slight sickness, headache continuing. Fairly good night; following morning head still aching, this continuing for about 24 hours. Third day: Swelling of valva much diminished. Mucous tubercles softer; ulceration on vulva much better, looked clean and healthy. Ulceration on nose quite red and cleaner gummata the same. Throat: tonsils less inflamed; swallowing not so painful; white patches on tonsils better Second injection of $0.15 \mathrm{gm}$. ; no reaction followed but a worse headache than usual for 24 hours. Fifth day: No swelling on vulva. Hard base of ulceration much softer. Ulceration healing; epidermisation could be seen growing from edges of sore. Ulceration of nose quite healed, flat with the skin. Mucous tubercles on vulva smaller. Gummata on scalp no longer discharging pus, looked clean and healthy. Throat much better; white ulcerated patches gone, tonsils smaller, swallowing not painful. Patient feeling stronger and better altogether. Third injection of $0.20 \mathrm{gm}$.; no reaction followed. Eighth day: Ulceration of vulva nearly healed, flat with skin; only a small area not epidermised. Mucous tubercles quite small and flat. Throat quite well, swallowing quite normal. Gummata on scalp getting smaller; quite dry, practically healed. Glands in groins and neck getting smaller still, and less hard. Patient quite well and gaining in weight. No headache. Fourth injection of $0.25 \mathrm{gm}$.; no reaction followed. Twelfth day: Scalp quite healed; glands still smaller. Patient quite well, wanted her discharge. Two further injections at regular intervals were given to complete treatment up to the total dose of $1 \cdot 20 \mathrm{gm}$. of luargol.

I have notes of one case with large papular syphilides of the face, arms, and legs, lesions which are most resistant to genuine German salvarsan. This case was treated with another arsenical substitute without improvement. I commenced treatment with luargol, after a complete course of injections of another substitute had failed to improve the condition. After four injections of $0.10 \mathrm{gm}$. every second day the lesions began to clear up and proceeded to improve until the twentieth day, when they had completely disappeared. The injections were increased at regular intervals till the total dose of $1.50 \mathrm{gm}$. was given to complete treatment.

Another case with hard pigmented papular general rash, lesions which take weeks and weeks to disappear with salvarsan, was also treated with another substitute without improvement at all. This case was put under luargol. One week after the beginning of the treatment the papules began to shrink and to flatten. Fifteen days afterwards most of them were flat with the skin and fading. In the third week all papules were quite flat and some quite faded. In several other cases all the symptoms cleared up after a single dose of $0.15 \mathrm{gm}$. of luargol, but it must be remembered that very often slight syphilitic symptoms 
disappear without any treatment at all. In other cases, after a dose of $0.05 \mathrm{gm}$. only, epidermisation began very quickly to grow from the edges of long-standing sores. I have received during the last month from several medical men who are using luargol in London, letters telling me that after injections of this remedy they met sometimes with very bad general reactions or with thrombosis or phlebitis of the veins. In the hundred cases treated here up to the present time, slight thrombosis followed the injections in four cases and this was due to the using of impure hydrate of sodium. Only chemically pure sodium should be used to dissolve luargol, otherwise, if there is a too large proportion of carbonate in the alkaline solution, luargol is converted into an insoluble black sediment of carbonate which on no account should be injected. If the proper solution of soda is employed, luargol gives a perfectly clear brown-black solution which hardly needs filtering, and no trouble whatever should follow the intravenous injection.

It is of the greatest importance that injections should not be made until the operator is assured his needle is in the vein. If in spite of this there is thrombosis the fact is due to an excess of soda in the alkaline solution. But I must add that thrombosis of the veins may occur with any other drug if, when injected into the vein, the solution is not isotonic. With each outfit of luargol a phial containing the correct diluted alkaline solution for the dose wanted is provided, and thus the risks of thrombosis are reduced to a minimum. In order to avoid this inconvenience Danysz has prepared lately a new preparation of this remedy, the sodic luargol, which dissolves simply in distilled water like neosalvarsan. I have used it now for two months with great satisfaction. This preparation is very handy, less toxic, and the efficacy as regards clinical results is exactly the same. It is not yet on the market, but will be provided in sealed tubes of $0 \cdot 10,0 \cdot 20$, $0.30,0.40$, and $0.50 \mathrm{gm}$. The solution is filtered before use for safety, and injected as before explained.

Regarding severe general reactions which may follow the intravenous injection, I had one case only. It was that of a girl of 24 years who had had three injections of luargol before, without any reaction afterwards. She got her fourth injection at the same time and with the same solution as 20 other patients. Alone, of these 20 cases, she complained, directly after the injection was made, of severe pains in the arm. There were congestion of the face, slight dyspnoea; she had a very high temperature up to $104^{\circ}$ three hours afterwards, with vomiting and headache. These symptoms lasted for 24 hours and the patient was quite well again and later had two more injections without any reaction afterwards.

Danysz showed that after intravenous injection of a product of the dioxydiaminoarsenobenzol group, symptoms of poisoning of very alarming aspect are sometimes observed ; congestion of the face, dyspnoea, urticaria, trembling and contraction or weakness of the limbs, convulsions, headaches, accompanied or not by temperatures of $102^{\circ}, 104^{\circ}$, and even $105^{\circ}$.

Struck by the resemblance of some of these symptoms to those of nitrite poisoning, M. Milian has given them the name of "nitritoid crises," and it is under this name that these accidents have since been generally described. These troubles always appear during or immediately after the injection, and no apparent symptom enables the practitioner to foresee the possibility of such an accident, generally more alarming than grave, which may arise in the case of individuals of the best constitution having all organs in perfect condition. Again, a nitritoid crisis may be observed in the case of one or two patients out of 10 or 15 who have received injections at the same time of the same product, prepared for all of them and taken from the same vessel; or the crisis may appear in the case of a patient who has already well borne one or several injections, and who may bear very well the subsequent ones. Some preliminary experiments, as well as numerous researches published on this question by Mouneyrat, Darier, Milian, Ravaut, \&c., the publications of Mr. Emery on the dangers of mineral impurities in the water used for the dissolution of arsenobenzol, and more especially the experimental researches of $\mathrm{Ch}$. Fleig on the toxicity of salvarsan, have led me to think that it should be possible to determine more exactly the conditions under which the whole of these troubles may arise and to ascertain the causes by a series of suitable experiments. These experiments bore in the first place on the manner in which a more or less alkalised solution of arsenobenzol or luargol acts in the presence of the salts which are ordinarily found in water and in the blood ; and, secondly, on the effect which these various preparations may have on the animal when injected into the veins. The following are the conclusions which were made therefrom :--

All monosodic or disndio produots of the arsenobenzol group become turbid and yield a preoipitate in the presenoe of chloride of sodium, carbonates, phosphates, sulphates, and chlorides of alkalies and magnesia, and more especially of phosphates. Some of these precipitates may re-dissolve in the same liquid, others remain insoluble even in an excess of soda. Their formation is prevented or retarded in certain cases in the presence of sugars, and rather accelerated in other cases (for instance, in the presence of glycerophosphate of lime). In general, a trace of these salts (1 or 2 drops of a 1 per cent. solution in 10 c.c.) will suffice to produce an appreciable effect.

If a rabbit is injected with a solution which becomes turbid in the physiological solution of pure sea salt in less than 10 minutes, a nitritoid crisis is always produced in the animal, which crisis will be fatal with a dose of $020 \mathrm{gm}$. very grave but not always fatal with a dose of $0.10 \mathrm{gm}$. very pronounced but not fatal (dyspncea, diarrhœa, conrulsive fits) with a dose of $0.05 \mathrm{gm}$. The last rabbit will, some hours afterwards, bear very well, without any crisis, a dose of $0.20 \mathrm{gm}$. of a solution which does not become turbid, in the presence of $\mathrm{NaCl}$ in the proportion of $8: 1000$, until after five or six hours. On the other hand, this latter solution will, if injected in the same dose, produce a fatal crisis in the case of a rabbit previously injected-one might say "rendered more susceptible"-with a few centigrammes of a phosphate of lime.

Ch. Fleig has found, in the necropsy of rabbits which had died from an acute nitritoid crisis, that precipitates of arsenobenzol were present in the capillaries of the lungs. Danysz has been able to corroborate this fact, so that his experiments enable him to assert with certainty that all the troubles which appear immediately or some hours after the injection are caused by the furmation of a preoipitate in the circulatory system.

It is scarcely necessary to point out here that the differences in the symptoms observed correspond to the differences in the nature of the precipitate and that these differences may themselves be determined by differences in the richness of the blood in certain salts (temporary or constant), in the intestinal mucous membrane, the skin, the kidneys, the brain, or the lungs. In the latter case carbonic acid may play an important part.

The whole of the troubles observed after injections of the products of the arsenobenzol group are also met with, identically the same, in the anaphylactic crisis, and it would be difficult to find a combination of experimental conditions more appropriate than those of the experiments just made with luargol, to prove that in an active or passive anaphylaotic orisis it is the precipitate whioh is the principal agent in the pathological manifestations. In both cases there are the same symptoms, the same causes (formation of precipitates in vitro and in vivo), the same means of avoiding the crises by anæsthetics or vaso-constrictors, as has been demonstrated by M. Roux with regard to anaphylactic and by $M$. Milian with regard to nitritoid crises.

\section{Conolusions.}

1. Thrombosis, phlebitis, and other local affections caused in the injected veins, if not due to faulty technique, may be attributed to excessive alkalisation of the solution.

2. Affections of a general nature,-vomiting, diarrhoea, shivering fits, headaches, transient rise of temperaturewhich may appear a few minutes or some hours after the injection, as well as the nitritoid crises, are caused by the dilatation of vessels and the formation of a precipitate which is temporarily retained in the capillaries.

3. The rapidity with which these crises appear, and the degree of their gravity, will depend on the dose of the product injected and its degree of concentration. By increasing the dose the volume of the precipitate is increased ; by increasing the concentration its formation is accelerated and the volume and density of the granules are augmented. Sufficient alkalisation of the products will obviate the appearance of nitritoid crises and other troubles in the case of individuals having 
blood of a normal average composition. To obviate the troubles caused by soda, the strength of which it is always difficult to maintain at the same percentage, it would be preferable to use chiefly disodic products prepared beforehand.

4. Assuming that the alkalisation is adequate, the formation of precipitates may be promoted by the presence in the solvents of sea-salt, and especially of phosphates, carbonates, sulphates, and chlorides of earthy alkalies and magnesia.

5. The formation of precipitates may be retarded, and the gravity of the nitritoid and anaphylactic crises thus diminished, by the following means: (a) By prolonging the duration of the injections and making them in the form of highly diluted solutions; $(b)$ by making the dilution with distilled water or isotonic solutions of sugar, which delay the formation of precipitates in most cases; and $(c)$ by injecting at the same time anæsthetics or vaso-constrictors.

6 . The neutral compounds of the products of the arsenobenzol group, which may be obtained by combination with methylene or formaldehyde sulphoxylate of sodium, neo or novo arsenobenzols, \&c., do not precipitate in the presence of chlorides, sulphates, and carbonates, but will do so in the presence of certain phosphates. These products will therefore not produce rapid crises save under exceptional conditions, but their use gives comparatively more often rise to tardy but graver accidents.

7. As regards the products of the arsenobenzol group, the formation of a harmless or not very injurious precipitate, which manifests itself by slight troubles in from one to six hours after the injection, is an essential pre-requisite of their therapeutic efficacy; time is an important factor in this instance. The sodic products are therefore more active than the neutral (nnvo) products, and when they are well prepared and injected with the necessary precautions. they are less dangerous than the neutral products. In fact, as in order to obtain the same curative effect we are obliged to inject the neutral products in two or three times stronger doses, they will, if retained in the organism through some cause or other and forming precipitates or toxic compounds, cause graver accidents than the sodic products.

8. It seems that under otherwise equal conditions the compounds which produce the same curative effects in relatively smaller doses will also be less dangerous to the patients.

9. It is too soon to say that " 102 " is the best remedy for syphilis, as we do not even know if salvarsan, "606," really cures this disease, though its great results in many cases are undisputed. But luargol is certainly a step forward in the therapy of syphilis, for it has proved its efficacy, since quite small, non-toxic and generally harmless doses give satisfactory clinical results.

\section{A NOTE ON THE USE OF PERFORATED CELLULOID IN THE DRESSING OF CERTAIN WOUNDS.}

By S. R. DOUGLAS, M.R.C.S., L.R.C.P. LOND.,

CAPTAIN, I.M.S. (RETTRED); TIRS' ASSISTANT, BACTERTOLOGICAL DEPARTMENT, MEDICAL RESEARCH COMMITTEE.

(From the Inoculation Department, st. Mary's Hospital, Paddington, W.)

THE problem of the alleviation of the pain caused by the removal of dressings which have become adherent to the wound is, at present, of more than usual importance owing to the types of wounds from which so many of the soldiers are at present suffering.

The special types referred to are those which either as a result of the primary injury, or of the operative measures necessitated by the onset of gas gangrene, have lost considerable areas of skin. In such cases the dressings are at times so painful as to act very detrimentally on the patients' general condition. Various methods were tried with the view of solving this problem, the principle of all being to place next the wound surface some non-absorbent material which, however, would allow free passage outwards of any discharges and inwards of any fluid that it was desired should reach the surface of the wound. After the trial of several materials, it was found that there were on the market sheets of perforated celluloid 0.15 millimetre in thickness, the perforations being 1.5 millimetres in diameter, and there being four perforations to each centimetre. This material, which was rather too stiff to be conveniently applied to the irregular surface of the wound, was found to become perfectly soft and pliable, and at the same time somewhat elastic, after it had been soaked in a 5 per cent. carbolic acid solution for a few hours. The carbolic acid solution having been washed away with sterile salt solution, the softened celluloid can be applied to the wound surface and falls at once into all the irregularities, and any suitable dressing can be applied over it. On redressing the wound it is found: (1) That the celluloid lifts off the surface of the wound without causing any pain; (2) that all the discharges from the wound have passed through the perforations, leaving the surface of the wound quite clean; and (3) that the celluloid has regained its original stifiness, thus making an accurately fitting splint, which tends to keep the wounded tissue in a complete state of rest. After the celluloid has been taken off the wound it is cleansed in tepid water and again softened and sterilised by placing it in the 5 per cent. carbolic acid solution. A convenient plan is to have two pieces of the celluloid for each wound, one of which is applied to the wounded area, the other being kept in a 5 per cent. solution of carbolic acid so as. to be ready for use.

This material can be obtained in varying thicknesses, the perforations being of the same size and number, and it has been found that the thicker sheets make an excellent material for the making of splints, especially for those cases for whom continuous or intermittent irrigation forms part of the treatment. For this purpose the sheets of perforated cellaloid, having been first softened in 5 per cent. carbolic acid solution, are moulded to the injured limb. After the celluloid has again hardened it is attached to a metal framework such as may be readily made with the aluminium skeleton splinting supplied in the field fracture-box.

It was at first thought that the wound granulations would tend to grow through the perforations, but this has occurred only in those cases in which the granulations tended to be œdematous, and in these the celluloid is not required, as ordinary gauze dressings showed no tendency to become adherent to the wound surface.

The material was obtained from the British Xylonite Company, Hale End, Walthamstow, London.

\section{Climital a}

MEDICAL, SURGICAL, OBSTETRICAL, AND THERAPEUTICAL.

\section{A CASE OF FOREIGN BODY IN THE RECTUM.}

\section{By Joseph F. Peart, F.R.C.S. Irel.}

I SEND for publication the following particulars, which are interesting as once more demonstrating the safety with which sharp-pointed foreign bodies may pass through the alimentary tract until arrested in a crypt or pocket of Morgagni, and possibly as indicating that wooden skewers should not be used in cooking food.

On May 23rd, 1915, I was called in to seen a married woman, aged 49 years, who was suffering from acute pain in the anal canal of 24 hours' duration. The pain was intense and prevented sleep or rest in any position, especially in any posture which occasioned any pressure upon the anus, such as sitting. The menstrual history was normal. The patient had first had an attack similar to the present one 15 years ago, when the practitioner who attended her examined her vaginally but could not detect any cause for the trouble, and the attack gradually passed off. No rectal examination was made. Eight years later she had another attack, though the pain was not so acute, and at varying intervals of time since then she had suffered from attacks of pain of lesser severity in the same situation. The bowels had been habitually costive, but for years she had kept them open daily with pills and did not remember having passed at any time blood or slime per anum.

There were no constitutional symptoms Abdominal and vaginal examination gave negative results. Externally 